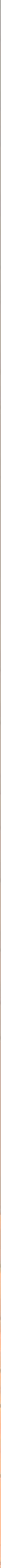




\title{
Heegner divisors in generalized Jacobians and traces of singular moduli
}

\author{
Jan Hendrik Bruinier and Yingkun Li
}

\begin{abstract}
We prove an abstract modularity result for classes of Heegner divisors in the generalized Jacobian of a modular curve associated to a cuspidal modulus. Extending the Gross-Kohnen-Zagier theorem, we prove that the generating series of these classes is a weakly holomorphic modular form of weight $\frac{3}{2}$. Moreover, we show that any harmonic Maass form of weight 0 defines a functional on the generalized Jacobian. Combining these results, we obtain a unifying framework and new proofs for the Gross-Kohnen-Zagier theorem and Zagier's modularity of traces of singular moduli, together with new geometric interpretations of the traces with nonpositive index.
\end{abstract}

\section{Introduction}

The celebrated Gross-Kohnen-Zagier theorem [Gross et al. 1987] states that the generating series of Heegner divisors on the modular curve $X_{0}(N)$ is a cusp form of weight $\frac{3}{2}$ with values in the Jacobian of $X_{0}(N)$. This result was later generalized by various authors to orthogonal and unitary Shimura varieties of higher dimension; see, e.g., [Borcherds 1999; Kudla 2004; Liu 2011].

In a different direction, Zagier [2002] proved that the traces of the normalized $j$-invariant over Heegner divisors of discriminant $-d$ on the modular curve $X(1)$ are the coefficients of a weakly holomorphic modular form of weight $\frac{3}{2}$. This result was also generalized in subsequent work to modular curves of arbitrary level, traces of harmonic Maass forms over twisted Heegner divisors, and to cover more general nonpositive weight modular functions; see, e.g., [Alfes and Ehlen 2013; Bringmann et al. 2005; Bruinier and Funke 2006; Duke and Jenkins 2008; Funke 2002; Kim 2004]. Recently, Gross [2012] has explained how Zagier's original result can be related to their earlier joint result with Kohnen. He showed that the traces of singular moduli on $X(1)$ can be interpreted in terms of Heegner divisors in the generalized Jacobian associated with the modulus $2 \cdot(\infty)$.

The authors are partially supported by DFG grant BR-2163/4-1.

MSC2010: primary 14G35; secondary 14H40, 11F27, 11F30.

Keywords: Singular moduli, generalized Jacobian, Heegner point, Borcherds product, harmonic Maass form. 
We pick up this idea of Gross and define classes of Heegner divisors of arbitrary discriminant in the generalized Jacobian $J_{\mathfrak{m}}(X)$ of a modular curve $X$ of arbitrary level with cuspidal modulus $\mathfrak{m}$. Then we prove that the generating series of these classes is a weakly holomorphic modular form of weight $\frac{3}{2}$ with values in $J_{\mathfrak{m}}(X)$. Our argument is a generalization of Borhcerds' proof [1999] of the Gross-KohnenZagier theorem [Borcherds 1999] and relies on the construction of explicit relations among Heegner divisors given by automorphic products. Note that, in contrast to [Borcherds 1999], we need to use the explicit infinite product expansions of automorphic products at all cusps of $X$. By applying the natural map between $J_{\mathfrak{m}}(X)$ and the usual Jacobian $J(X)$ to this generating series we recover the "classical" Gross-Kohnen-Zagier theorem.

Then we show that every harmonic Maass form $F$ of weight 0 on $X$ with vanishing constant term at every cusp (such as the normalized $j$-function when $X=X(1)$ ) defines a functional $\operatorname{tr}_{F}$ on $J_{\mathfrak{m}}(X)$. The value of $\operatorname{tr}_{F}$ on Heegner divisors of negative discriminant $-d$ is just the sum of the values of $F$ over the Heegner points of discriminant $-d$. The value of $\operatorname{tr}_{F}$ on "Heegner divisors" of nonnegative discriminant can be explicitly computed in terms of the principal parts of $F$ at the cusps. In that way we are able to recover Zagier's result and its generalizations in [Alfes and Ehlen 2013; Bruinier and Funke 2006].

We now describe the content of the present paper in more detail. To simplify the exposition, throughout this introduction we let $p$ be prime or 1 and consider the modular curve $X_{0}^{*}(p)$ associated to the extension $\Gamma_{0}^{*}(p)$ of $\Gamma_{0}(p)$ in $\operatorname{PSL}_{2}(\mathbb{Z})$ by the Fricke involution. In the body of this paper, we consider modular curves of arbitrary level (as modular curves associated to orthogonal groups of signature $(1,2)$ ).

Let $\infty$ be the cusp of $X_{0}^{*}(p)$ and let $m$ be a nonnegative integer. Then $\mathfrak{m}=m \cdot(\infty)$ is an effective divisor. Recall that the generalized Jacobian $J_{\mathfrak{m}}\left(X_{0}^{*}(p)\right)$ of $X_{0}^{*}(p)$ associated with the modulus $\mathfrak{m}$ is a commutative algebraic group whose rational points correspond to classes of divisors of degree zero modulo $\mathfrak{m}$-equivalence; see Section 2 and [Serre 1988]. If $m=0$, then $J_{\mathfrak{m}}\left(X_{0}^{*}(p)\right)$ is simply the usual Jacobian. For any integer $d$, let $\mathcal{Q}_{p, d}$ be the set of (positive definite if $d>0$ ) integral binary quadratic forms $[a, b, c]$ of discriminant $-d=b^{2}-4 a c$ with $p$ dividing $a$. If $d \neq 0$ then $\Gamma_{0}^{*}(p)$ acts on $\mathcal{Q}_{p, d}$ with finitely many orbits.

If $d$ is positive, then any $Q \in \mathcal{Q}_{p, d}$ defines a point $\alpha_{Q}$ in the upper complex half-plane $\mathbb{H}$, the solution of the equation $a z^{2}+b z+c=0$ with positive imaginary part. There is a corresponding Heegner divisor of discriminant $-d$ on $X_{0}^{*}(p)$ given by

$$
Y(d)=\sum_{Q \in \mathcal{Q}_{p, d} / \Gamma_{0}^{*}(p)} \frac{1}{\left|\Gamma_{0}^{*}(p)_{Q}\right|} \cdot\left(\alpha_{Q}\right),
$$

where $\Gamma_{0}^{*}(p)_{Q}$ is the (finite) stabilizer of $Q$ (see Equation (1.5) in [Bruinier and 
Funke 2006]). The divisor

$$
Z(d)=Y(d)-\operatorname{deg}(Y(d)) \cdot(\infty)
$$

has degree zero and is defined over $\mathbb{Q}$. We denote by $[Z(d)]_{\mathfrak{m}}$ its class in the generalized Jacobian $J_{\mathfrak{m}}\left(X_{0}^{*}(p)\right)$.

If $d$ is negative, any $Q \in \mathcal{Q}_{p, d}$ defines an oriented geodesic cycle on $\mathbb{U} \cup P^{1}(\mathbb{R})$, given by the equation $a|z|^{2}+b \Re(z)+c=0$. It has nontrivial intersection with $P^{1}(\mathbb{Q})$ if and only if $d$ is the negative of a square of an integer. In this case the two solutions in $P^{1}(\mathbb{Q})$ define cusps of the modular curve. There is a unique cusp $c_{Q} \in P^{1}(\mathbb{Q})$ from which the geodesic originates. (In the present $\Gamma_{0}^{*}(p)$ example all cusps collapse to $\infty$ under the map to the quotient, but this is of course not true for more general congruence subgroups.) If $d=-b^{2}$ for a nonzero integer $b$, then $Q$ is $\Gamma_{0}^{*}(p)$-equivalent to $[0, b, c]$ with $c \in \mathbb{Z} / b \mathbb{Z}$ and $c_{Q}$ is equivalent to $\infty$. We let $h_{Q} \in \mathbb{Q}\left(X_{0}^{*}(p)\right)^{\times}$be a function satisfying

$$
h_{Q}=1-q_{\infty}^{b}+O\left(q_{\infty}^{m}\right)
$$

at the cusp $\infty$, where $q_{\infty}$ is the uniformizing parameter of the completed local ring at $\infty$ given by the Tate curve over $\mathbb{Z} \llbracket q_{\infty} \rrbracket$. Then we define

$$
[Z(d)]_{\mathfrak{m}}=\left[\operatorname{div}\left(h_{[0, b, 0]}\right)\right]_{\mathfrak{m}}=\sum_{Q \in \mathcal{Q}_{p, d} / \Gamma_{0}^{*}(p)} \frac{1}{b} \cdot\left[\operatorname{div}\left(h_{Q}\right)\right]_{\mathfrak{m}} .
$$

Note that this class vanishes if $d \leq-m^{2}$. If $d<0$ is not the negative of the square of an integer, we put $[Z(d)]_{\mathfrak{m}}=0$. Finally, for $d=0$, we define $[Z(0)]_{\mathfrak{m}}$ as the class of the line bundle of modular forms $\mathcal{M}_{-1}$ of weight -1 on $X_{0}^{*}(p)$ (see Section 2 for details).

To describe the relations among the classes $[Z(d)]_{\mathfrak{m}}$, we consider the generating series

$$
A_{\mathfrak{m}}(\tau)=\sum_{\substack{d \in \mathbb{Z} \\ d>-m^{2}}}[Z(d)]_{\mathfrak{m}} \cdot q^{d} \in \mathbb{C}((q)) \otimes J_{\mathfrak{m}}\left(X_{0}^{*}(p)\right) .
$$

It is a formal Laurent series in the variable $q=e^{2 \pi i \tau}$ for $\tau \in \mathbb{H}$. Our first main result is the following (see also Theorem 4.2).

Theorem 1.1. The generating series $A_{\mathfrak{m}}(\tau)$ is a weakly holomorphic modular form of weight $\frac{3}{2}$ for the group $\Gamma_{0}(4 p)$, that is, $A_{\mathfrak{m}}(\tau) \in M_{3 / 2}^{!}\left(\Gamma_{0}(4 p)\right) \otimes J_{\mathfrak{m}}\left(X_{0}^{*}(p)\right)$.

Under the natural map

$$
J_{\mathfrak{m}}\left(X_{0}^{*}(p)\right) \rightarrow J\left(X_{0}^{*}(p)\right)
$$

the classes $[Z(d)]_{\mathfrak{m}}$ with $d \leq 0$ are mapped to zero. Applying it to $A_{\mathfrak{m}}(\tau)$, we recover the Gross-Kohnen-Zagier theorem (see also Corollary 4.5). 
Corollary 1.2 (Gross-Kohnen-Zagier). The generating series $A_{0}(\tau)$ of classes of Heegner divisors $[Z(d)]_{0}$ in the Jacobian is a cusp form of weight $\frac{3}{2}$ for the group $\Gamma_{0}(4 p)$, that is, $A_{0}(\tau) \in S_{3 / 2}\left(\Gamma_{0}(4 p)\right) \otimes J\left(X_{0}^{*}(p)\right)$.

To recover the results of [Zagier 2002] and [Bruinier and Funke 2006] on traces of modular functions from Theorem 1.1, we show that harmonic Maass forms define functionals on $J_{\mathfrak{m}}\left(X_{0}^{*}(p)\right)$. Let $F \in H_{0}^{+}\left(\Gamma_{0}^{*}(p)\right)$ be a harmonic Maass form for $\Gamma_{0}^{*}(p)$ of weight 0 as in [Bruinier and Funke 2004]. Denote the Fourier expansion of the holomorphic part of $F$ by

$$
F^{+}(\tau)=\sum_{n \gg-\infty} c_{F}^{+}(n) \cdot q_{\infty}^{n}
$$

Proposition 1.3. Assume that $c_{F}^{+}(n)=0$ for $n \leq-m$ and $c_{F}^{+}(0)=0$. Then there is a linear map $\operatorname{tr}_{F}: J_{\mathfrak{m}}\left(X_{0}^{*}(p)\right) \rightarrow \mathbb{C}$ defined by

$$
[D]_{\mathfrak{m}} \mapsto \operatorname{tr}_{F}(D):=\sum_{a \in \operatorname{supp}(D) \backslash\{\infty\}} n_{a} \cdot F(a),
$$

for divisors $D=\sum_{a} n_{a} \cdot(a)$ in $\operatorname{Div}^{0}\left(X_{0}^{*}(p)\right)$.

The images under $\operatorname{tr}_{F}$ of the classes $[Z(d)]_{\mathfrak{m}}$ with $d \leq 0$ can be explicitly computed in terms of the principal part of $F$. As a consequence we derive the following theorem (see also Theorem 5.2).

Theorem 1.4. The series $\operatorname{tr}_{F}\left(A_{\mathfrak{m}}\right)$ is a weakly holomorphic modular form in the space $M_{3 / 2}^{!}\left(\Gamma_{0}(4 p)\right)$. It is explicitly given by

$$
\begin{aligned}
\operatorname{tr}_{F}\left(A_{\mathfrak{m}}\right)=\sum_{d>0} F(Y(d)) \cdot q^{d}+\sum_{n \geq 1} c_{F}^{+}(-n)\left(\sigma_{1}(n)+\right. & \left.p \sigma_{1}(n / p)\right) \\
& -\sum_{b>0} \sum_{n>0} c_{F}^{+}(-b n) \cdot b \cdot q^{-b^{2}} .
\end{aligned}
$$

The modularity of the right-hand side was also proved in [Bruinier and Funke 2006] by interpreting it as the Kudla-Millson theta lift of $F$. Applying this theorem to the special case where $p=1, m \geq 2$, and $F=j-744$, Zagier's original result on traces of singular moduli can be obtained.

In the body of the paper we work with modular curves of arbitrary level associated with orthogonal groups of even lattices of signature $(1,2)$. This setup is natural, since the proof of Theorem 1.1 implicitly relies on the singular theta correspondence for the dual reductive pair given by $\mathrm{SL}_{2}$ and $\mathrm{O}(1,2)$. For the modulus we allow arbitrary effective divisors that are supported on the cusps. The generating series of Heegner divisors is then a vector-valued modular form for the metaplectic extension of $\mathrm{SL}_{2}(\mathbb{Z})$ transforming with the Weil representation of a finite quadratic module. 
In Section 2 we recall some basic facts on generalized Jacobians of curves. Section 3 contains our setup for modular curves associated to orthogonal groups, Heegner divisors, and vector-valued modular forms. Then we define classes of Heegner divisors in generalized Jacobians in Section 4, and prove the abstract modularity theorem for these classes. In Section 5 we prove that harmonic Maass forms define functionals on the generalized Jacobian and derive modularity results for the traces of harmonic Maass forms over Heegner divisors from the abstract modularity theorem. We also give some explicit examples and indicate possible generalizations in Section 6.

\section{Generalized Jacobians}

Let $X$ be a complete nonsingular algebraic curve over a field $k$ of characteristic 0 . Let $\operatorname{Div}^{0}(X)$ be the group of divisors of $X$ of degree 0 defined over $k$, and denote by $P(X)$ the subgroup of divisors of rational functions $f \in k(X)^{\times}$. The Jacobian $J(X)$ of $X$ is a commutative algebraic group over $k$ whose $k$-rational points are isomorphic to the quotient group $\operatorname{Div}^{0}(X) / P(X)$.

Recall that there is the notion of the generalized Jacobian; see, e.g., [Serre 1988, Chapter 5] for details. Let $S \subset X(k)$ be a finite set of points, and for $s \in S$ let $m_{s} \in \mathbb{Z}_{\geq 0}$. Then

$$
\mathfrak{m}=\sum_{s \in S} m_{s} \cdot(s)
$$

is an effective divisor defined over $k$. Let $\mathcal{O}_{s}$ be the ring of integers in the completion $k(X)_{s}$ of $k(X)$ at $s$, and let $\pi_{s} \in \mathcal{O}_{s}$ be a uniformizer. If $f, g \in k(X)_{s}$ and $n \in \mathbb{Z}$, we write

$$
f=g+O\left(\pi_{s}^{n}\right)
$$

if $f-g \in \pi_{s}^{n} \mathcal{O}_{s}$. We consider the subgroup

$$
P_{\mathfrak{m}}(X)=\left\{\operatorname{div}(f): f \in k(X)^{\times} \text {with } \pi_{s}^{-\operatorname{ord}_{s}(f)} f=1+O\left(\pi_{s}^{m_{s}}\right) \text { for all } s \in S\right\}
$$

of $P(X)$. The generalized Jacobian $J_{\mathfrak{m}}(X)$ associated with the modulus $\mathfrak{m}$ is a commutative algebraic group over $k$, whose $k$-rational points satisfy

$$
J_{\mathfrak{m}}(X)(k) \cong \operatorname{Div}^{0}(X) / P_{\mathfrak{m}}(X) .
$$

The quotient on the right hand side is also canonically isomorphic to the subgroup of divisors in $\operatorname{Div}^{0}(X)$ coprime to $S$ modulo m-equivalence. For a divisor $D \in \operatorname{Div}^{0}(X)$ we denote by $[D]_{\mathfrak{m}}$ the corresponding class in $J_{\mathfrak{m}}(X)(k)$.

There is a canonical rational map $\varphi_{\mathfrak{m}}: X \rightarrow J_{\mathfrak{m}}(X)$ defined over $k$ which is regular outside $S$, see [Serre 1988, Chapter 5, Theorem 1]. If $\mathfrak{m}^{\prime}$ is another effective divisor on $X$ satisfying $\mathfrak{m} \geq \mathfrak{m}^{\prime} \geq 0$, there exists a unique homomorphism $J_{\mathfrak{m}} \rightarrow J_{\mathfrak{m}^{\prime}}$ 
which is compatible with $\varphi_{\mathfrak{m}}$ and $\varphi_{\mathfrak{m}^{\prime}}$. It is surjective and separable [Serre 1988, Chapter 5, Proposition 6]. In particular, there exists a surjective homomorphism

$$
J_{\mathfrak{m}}(X) \rightarrow J(X) .
$$

Its kernel is isomorphic to

$$
H_{\mathfrak{m}}=\left(\prod_{\substack{s \in S \\ m_{s}>0}} \mathbb{G}_{m} \times \mathbb{G}_{a}^{m_{s}-1}\right) / \mathbb{G}_{m},
$$

where the quotient is with respect to the diagonally embedded multiplicative group. Typical elements of the kernel are obtained, by choosing a pair $(s, n)$ with $s \in S$ and $n>0$ and a function $h_{s, n} \in k(X)^{\times}$such that

$$
h_{s, n}= \begin{cases}1-\pi_{s}^{n}+O\left(\pi_{s}^{m_{s}}\right) & \text { at } s, \\ 1+O\left(\pi_{t}^{m_{t}}\right) & \text { at all } t \in S \backslash\{s\} .\end{cases}
$$

An argument as in [Serre 1988, Chapter 5, Proposition 8] shows that the "additive part" of $H_{\mathfrak{m}}$ is generated by the classes

$$
\left[\operatorname{div}\left(h_{s, n}\right)\right]_{\mathfrak{m}},
$$

for $s \in S$ and $0<n<m_{s}$. Note that for $n \geq m_{s}$ the class $\left[\operatorname{div}\left(h_{s, n}\right)\right]_{\mathfrak{m}}$ vanishes.

Let $s_{0} \in S$ be a fixed base point. If $\mathcal{L}$ is a line bundle on $X$ which is defined over $k$, and $\left(\phi_{s}\right)_{s \in S}$ is a family of local trivializations of $\mathcal{L}$ at the points of $S$, we can associate to the pair $\left(\mathcal{L},\left(\phi_{s}\right)\right)$ a class in $J_{\mathfrak{m}}(X)$ as follows. It is easily seen that there exists a rational section $f$ of $\mathcal{L}$ such that

$$
\phi_{s}^{-1} f=\pi_{s}^{a_{s}} \cdot\left(1+O\left(\pi_{s}^{m_{s}}\right)\right),
$$

for some $a_{s} \in \mathbb{Z}$ at every $s \in S$. Then we define

$$
\left[\left(\mathcal{L},\left(\phi_{s}\right)\right)\right]_{\mathfrak{m}}=\left[\operatorname{div}(f)-\operatorname{deg}(\mathcal{L}) \cdot\left(s_{0}\right)\right]_{\mathfrak{m}} \in J_{\mathfrak{m}}(X)(k) .
$$

\section{Modular curves}

Here we recall the description of modular curves as Shimura varieties associated to orthogonal groups. We also define classes of Heegner divisors in generalized Jacobians.

Let $(L, Q)$ be an isotropic even lattice of signature $(1,2)$. We denote by $(x, y)$ the bilinear form corresponding to the quadratic form $Q$, normalized such that $Q(x)=\frac{1}{2}(x, x)$. For any commutative $\operatorname{ring} R$ we write $L_{R}=L \otimes_{\mathbb{Z}} R$. Throughout we fix an orientation on $L_{\mathbb{R}}$, and write $L^{\prime}$ for the dual lattice of $L$. Let

$$
N=\min \left\{n \in \mathbb{Z}_{>0}: n Q(\lambda) \in \mathbb{Z} \text { for all } \lambda \in L^{\prime}\right\}
$$


be the level of $L$, and denote by $\operatorname{disc}(L)=\left|L^{\prime} / L\right|$ the discriminant of $L$. We let $\mathrm{SO}(L)$ be the special orthogonal group of $L$ and write $\mathrm{SO}^{+}(L)$ for the intersection of $\mathrm{SO}(L)$ with the connected component of the identity of $\mathrm{SO}(L)(\mathbb{R})$. The even Clifford algebra of $L_{\mathbb{Q}}$ is isomorphic to the matrix algebra $\operatorname{Mat}_{2}(\mathbb{Q})$, which induces an isomorphism $\mathrm{PGL}_{2}(\mathbb{Q}) \cong \mathrm{SO}(L)(\mathbb{Q})$. We realize the hermitian symmetric space corresponding to $\mathrm{SO}(L)$ as the domain

$$
\mathcal{D}=\left\{z \in L_{\mathbb{C}}:(z, z)=0,(z, \bar{z})<0\right\} / \mathbb{C}^{\times} .
$$

It decomposes into 2 connected components. We fix one of these components and denote it by $\mathcal{D}^{+}$.

Let $\Gamma=\Gamma_{L}$ be the discriminant kernel subgroup of $\mathrm{SO}^{+}(L)$, that is, the kernel of the natural homomorphism

$$
\mathrm{SO}^{+}(L) \rightarrow \operatorname{Aut}\left(L^{\prime} / L\right) .
$$

Recall that rescaling the quadratic form by a factor of $n$ does not change $\mathrm{SO}^{+}(L)$ while it replaces the discriminant kernel by the full congruence subgroup of level $n$. We denote by

$$
Y_{\Gamma}=\Gamma \backslash \mathcal{D}^{+}
$$

the noncompact modular curve associated with $\Gamma$.

Let $\operatorname{Iso}(L)$ be the set of isotropic lines in $L$ (i.e., primitive isotropic rank-1 sublattices $I \subset L)$. The group $\Gamma$ acts with finitely many orbits on $\operatorname{Iso}(L)$. We denote by $X_{\Gamma}$ the compact modular curve obtained by adding to $Y_{\Gamma}$ the cusps corresponding to the $\Gamma$-classes of isotropic lines $I \in \operatorname{Iso}(L)$; see, e.g., [Bruinier and Funke 2006]. It is well known that $X_{\Gamma}$ is a projective algebraic curve which has a canonical model over a cyclotomic field.

As in [Bruinier and Funke 2006], we choose an orientation on the isotropic lines as follows. We fix one line $I_{0} \in \operatorname{Iso}(L)$ together with an orientation on $I_{0}$ given by a basis vector $x_{0} \in I_{0, \mathbb{R}}$. For any other $I \in \operatorname{Iso}(L)$ we choose a $g \in \mathrm{SO}^{+}(L)(\mathbb{R})$ such that $g I_{0, \mathbb{R}}=I_{\mathbb{R}}$. Then $g x_{0} \in I_{\mathbb{R}}$ defines an orientation on $I$ which is independent of the choices of $g$ and $x_{0}$.

Let $I \subset L$ be a primitive isotropic line and write $c_{I} \in X_{\Gamma}$ for the cusp corresponding to $I$. Local coordinates near $c_{I}$ can be described as follows. We write $N_{I}$ for the positive generator of the ideal $(I, L) \subset \mathbb{Z}$. It is a divisor of $N$. Throughout, we let $\ell=\ell_{I}$ be the positive generator of $I$ and fix a vector $\ell^{\prime}=\ell_{I}^{\prime} \in L^{\prime}$ such that

$$
\left(\ell, \ell^{\prime}\right)=1 \text {. }
$$

We let $K$ be the even negative definite lattice

$$
K=L \cap \ell^{\perp} \cap \ell^{\perp} .
$$


If $\ell_{K} \in K$ denotes a generator, then $K$ is isomorphic to $\mathbb{Z}$ equipped with the quadratic form $x \mapsto Q\left(\ell_{K}\right) x^{2}$. The quantity $4 Q\left(\ell_{K}\right)$ divides $N$. The holomorphic map

$$
\mathbb{H} \rightarrow \mathcal{D}, \quad w \mapsto \mathbb{C}^{\times}\left(w \otimes \ell_{K}+\ell^{\prime}-Q\left(w \otimes \ell_{K}\right) \ell-Q\left(\ell^{\prime}\right) \ell\right)
$$

is injective and has one of the two connected components of $\mathcal{D}$ as its image. Possibly replacing $\ell_{K}$ by its negative, we may assume that this map is an isomorphism from $\mathbb{Z}$ onto $\mathcal{D}^{+}$. It is compatible with the natural actions of $\mathrm{PGL}_{2}^{+}(\mathbb{Q})$ on $\mathbb{H}$ by fractional linear transformations and on $\mathcal{D}^{+}$via the isomorphism with $\mathrm{SO}^{+}(L)(\mathbb{Q})$. For $\mu \in L_{\mathbb{Q}} \cap I^{\perp}$ we consider the Eichler transformation

$$
E_{\ell, \mu}(x)=x+(x, \ell) \mu-(x, \mu) \ell-(x, \ell) Q(\mu) \ell
$$

in $\mathrm{SO}^{+}(L)(\mathbb{Q})$. It belongs to $\Gamma$ if $\mu \in K$.

Lemma 3.1. The stabilizer in $\Gamma$ of the primitive isotropic line I is given by

$$
\Gamma_{I}=\left\{E_{\ell, \mu}: \mu \in K\right\} .
$$

Proof. Let $\gamma \in \Gamma_{I}$. Then $\gamma \ell= \pm \ell$. We first assume that $\gamma \ell=\ell$. Then

$$
u:=\gamma \ell^{\prime}-\ell^{\prime}
$$

belongs to $L \cap \ell^{\perp}$, and $v:=u-\left(u, \ell^{\prime}\right) \ell$ belongs to $K$. It is easily checked that

$$
E_{\ell, v}(\ell)=\ell, \quad E_{\ell, v}\left(\ell^{\prime}\right)=\gamma \ell^{\prime} .
$$

Hence $\gamma^{-1} E_{\ell, v}$ leaves the vectors $\ell$ and $\ell^{\prime}$ fixed. Consequently, it maps the orthogonal complement $K$ to itself, and therefore $\ell_{K}$ to $\pm \ell_{K}$. Since $\gamma^{-1} E_{\ell, v}$ has determinant 1, the sign must be positive and thus $\gamma=E_{\ell, v}$.

We now consider the case $\gamma \ell=-\ell$. The orthogonal transformation $\sigma$ taking $\ell$ to $-\ell$, and $\ell^{\prime}$ to $-\ell^{\prime}$, and $\ell_{K}$ to itself belongs to $\operatorname{SO}(L)(\mathbb{Q})$. The element $\sigma \gamma \in \mathrm{SO}(L)(\mathbb{Q})$ fixes $\ell$. Arguing as above, we see that it is equal to an Eichler transformation $E_{\ell, u} \in \mathrm{SO}^{+}(L)(\mathbb{Q})$. This implies that $\sigma$ belongs to the connected component of the identity of $\mathrm{SO}(L)(\mathbb{R})$. But this leads to a contradiction, since the spinor norm of $\sigma$ is negative, showing that the case $\gamma \ell=-\ell$ cannot occur.

The action of $\mathbb{Z}$ on $\mathbb{U}$ by translations corresponds to the action of $\Gamma_{I}$ on $\mathcal{D}^{+}$. The induced map

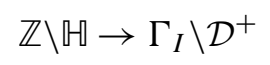

is an isomorphism. Hence, $q_{I}=e^{2 \pi i w}$ defines a local parameter at the cusp $c_{I}$ of $X_{\Gamma}$.

Example 3.2. In the special case when $N_{I}=1$, then $4 N=-Q\left(\ell_{K}\right)$ and the discriminant kernel subgroup $\Gamma$ is isomorphic to $\Gamma_{0}(N / 4)$. The curve $X_{\Gamma}$ is isomorphic to $X_{0}(N / 4)$, with $c_{I}$ corresponding to the cusp at $\infty$; see, e.g., [Bruinier and Ono 2010, Section 2.4]. 
The Weil representation. Let $\mathrm{Mp}_{2}(\mathbb{Z})$ be the metaplectic extension of $\mathrm{SL}_{2}(\mathbb{Z})$ by $\{ \pm 1\}$, realized by the two possible choices of a holomorphic square root of the automorphy factor $c \tau+d$ for $\left(\begin{array}{ll}a & b \\ c & d\end{array}\right) \in \mathrm{SL}_{2}(\mathbb{Z})$; see, e.g., [Borcherds 1998; Kudla 2003].

Recall that there is a Weil representation $\omega_{L}$ of $\mathrm{Mp}_{2}(\mathbb{Z})$ on the complex vector space $S_{L}$ of functions $L^{\prime} / L \rightarrow \mathbb{C}$ on the discriminant group. Identifying $S_{L}$ with the space of Schwartz-Bruhat functions on $L \otimes \hat{\mathbb{Q}}$ which are supported on $L^{\prime} \otimes \hat{\mathbb{Z}}$ and translation invariant under $L \otimes \hat{\mathbb{Z}}$, the representation $\omega_{L}$ can be viewed as the restriction of the usual Weil representation of $\mathrm{Mp}_{2}(\hat{\mathbb{Q}})$ on $L \otimes \hat{\mathbb{Q}}$ with respect to the standard additive character of $\hat{\mathbb{Q}}$; see [Kudla 2003]. The representation $\omega_{L}$ is the complex conjugate of the representation $\rho_{L}$ in [Borcherds 1998; Bruinier 2002; Bruinier and Funke 2006]. The action of $\mathrm{Mp}_{2}(\mathbb{Z})$ on $S_{L}$ commutes with the natural action of $\operatorname{Aut}\left(L^{\prime} / L\right)$ by translation of the argument.

If $k \in \frac{1}{2} \mathbb{Z}$, we denote by $M_{k}^{!}\left(\omega_{L}\right)$ the space of $S_{L}$-valued weakly holomorphic modular forms for $\operatorname{Mp}_{2}(\mathbb{Z})$ of weight $k$ with representation $\omega_{L}$. The subspace of holomorphic modular forms is denoted by $M_{k}\left(\omega_{L}\right)$.

Heegner divisors. For any $d \in \mathbb{Q}^{\times}$, the group $\Gamma$ acts on the set

$$
L_{d}^{\prime}=\left\{\lambda \in L^{\prime}: Q(\lambda)=d\right\}
$$

with finitely many orbits. For every $\lambda \in L^{\prime}$ with $Q(\lambda)>0$, the stabilizer $\Gamma_{\lambda} \subset \Gamma$ of $\lambda$ is finite, and there is a unique point $z_{\lambda} \in \mathcal{D}^{+}$which is orthogonal to $\lambda$. For $d \in \mathbb{Q}_{>0}$ and $\varphi \in S_{L}$ we consider the Heegner divisor

$$
Y(d, \varphi)=\sum_{\lambda \in L_{d}^{\prime} / \Gamma} \frac{1}{2\left|\Gamma_{\lambda}\right|} \varphi(\lambda) \cdot\left(z_{\lambda}\right)
$$

on $X_{\Gamma}$. It is defined over the field of definition of $X_{\Gamma}$ and has coefficients in the field of definition of $\varphi$. Let $I_{0} \in \operatorname{Iso}(L)$ be a fixed isotropic line. We define a divisor of degree 0 on $X_{\Gamma}$ by putting

$$
Z(d, \varphi)=Y(d, \varphi)-\operatorname{deg}(Y(d, \varphi)) \cdot\left(c_{I_{0}}\right) .
$$

\section{A generalized Gross-Kohnen-Zagier theorem}

We now consider classes of Heegner divisors in the generalized Jacobian of the modular curve $X:=X_{\Gamma}$ as defined in the previous section. We let $k \subset \mathbb{C}$ be the number field obtained by adjoining the primitive root of unity $e^{2 \pi i / N}$ to the common field of definition of the canonical model and all of the cusps of $X$. Let $S=\left\{c_{I}: I \in \operatorname{Iso}(L) / \Gamma\right\}$ be the set of cusps of $X$ and let

$$
\mathfrak{m}=\sum_{I \in \operatorname{Iso}(L) / \Gamma} m_{I} \cdot\left(c_{I}\right)
$$


be a fixed effective divisor supported on $S$. We consider the generalized Jacobian of $X$ associated with the modulus $\mathfrak{m}$. For $I \in \operatorname{Iso}(L)$, we take as the uniformizing parameter in the completed local ring at $c_{I}$ the parameter $q_{I}=e^{2 \pi i w}$ defined by (3-6) (given by the Tate curve over $\mathbb{Z} \llbracket q_{I} \rrbracket$ when $N_{I}=1$ such that $X_{\Gamma} \cong X_{0}(N / 4)$ ).

Since, throughout this section, we are only interested in the $k$-valued points of the generalized Jacobian, we briefly write $J_{\mathfrak{m}}(X)$ instead of $J_{\mathfrak{m}}(X)(k)$. For every degree-zero divisor $D=\sum a_{I} \cdot\left(c_{I}\right) \in \operatorname{Div}^{0}(X)$ supported on $S$ and every tuple $r=\left(r_{I}\right) \in \mathbb{G}_{m}^{|S|}(k)$, we choose a function $u_{D, r} \in k(X)^{\times}$such that

$$
u_{D, r}=r_{I} q_{I}^{a_{I}} \cdot\left(1+O\left(q_{I}^{m_{I}}\right)\right)
$$

at $c_{I}$ for $I \in \operatorname{Iso}(L)$. We write $H_{\mathbb{G}_{m}, \mathfrak{m}}$ for the subgroup of $J_{\mathfrak{m}}(X)$ generated by the classes $\left[\operatorname{div}\left(u_{D, r}\right)\right]_{\mathfrak{m}}$ of all these functions and let

$$
J_{\mathfrak{m}}^{\text {add }}(X)=J_{\mathfrak{m}}(X) / H_{\mathbb{G}_{m}}, \mathfrak{m} .
$$

By definition we have $J_{\mathfrak{m}}^{\text {add }}(X)=J_{\mathfrak{m}}(X)$ when $|S|=1$. By the Manin-Drinfeld theorem we have $J_{\mathfrak{m}}^{\text {add }}(X)_{\mathbb{Q}}=J_{\mathfrak{m}}(X)_{\mathbb{Q}}$ when $\mathfrak{m}=0$. For general $\mathfrak{m}$ the kernel of the induced homomorphism

$$
J_{\mathfrak{m}}^{\text {add }}(X)_{\mathbb{Q}} \rightarrow J(X)_{\mathbb{Q}}
$$

is isomorphic to the product of the groups $\mathbb{G}_{a}^{m_{I}-1}$ for $I \in \operatorname{Iso}(L) / \Gamma$ with $m_{I}>0$.

For $d \in \mathbb{Q}_{>0}$ and $\varphi \in S_{L}$ we consider the class

$$
[Z(d, \varphi)]_{\mathfrak{m}} \in J_{\mathfrak{m}}(X)_{\mathbb{C}}
$$

of the Heegner divisor $Z(d, \varphi)$ in the generalized Jacobian.

Let $\mathcal{T}$ be the tautological bundle on $X$, and define the line bundle of modular forms of weight $2 k$ on $X$ by $\mathcal{M}_{2 k}=\mathcal{T}^{\otimes k}$. (Sections of $\mathcal{M}_{2 k}$ correspond to classical elliptic modular forms of weight $2 k$ under the isomorphism $\mathrm{SO}(L)(\mathbb{Q}) \cong \mathrm{PGL}_{2}(\mathbb{Q})$.) Recall that $\mathcal{T}$ is canonically trivial in small neighborhoods of the cusps. Hence, taking the induced trivializations and putting $s_{0}=c_{I_{0}}$ in (2-7), we obtain a class $\left[\mathcal{M}_{k}\right]_{\mathfrak{m}} \in J_{\mathfrak{m}}(X)_{\mathbb{Q}}$. For $d=0$ we define

$$
[Z(0, \varphi)]_{\mathfrak{m}}=\varphi(0) \cdot\left[\mathcal{M}_{-1}\right]_{\mathfrak{m}} .
$$

We also define classes for $d \in \mathbb{Q}_{<0}$ as follows. For a vector $\lambda \in L_{d}^{\prime}$, the orthogonal complement $\lambda^{\perp} \subset L_{\mathbb{Q}}$ is isotropic if and only if $d \in-2 \operatorname{disc}(L)\left(\mathbb{Q}^{\times}\right)^{2}$. In this case there is a unique pair of isotropic lines $I, \tilde{I} \in \operatorname{Iso}(L)$ such that $\lambda^{\perp}=I_{\mathbb{Q}} \oplus \tilde{I}_{\mathbb{Q}}$ and such that the triple $(\lambda, x, \tilde{x})$ is a positively oriented basis of $L_{\mathbb{Q}}$ for positive basis vectors $x \in I$ and $\tilde{x} \in \tilde{I}$. Following [Bruinier and Funke 2006], we call $I$ the isotropic line associated to $\lambda$ and write $I \sim \lambda$. Note that $\tilde{I}$ is the isotropic line associated to $-\lambda$. We define the $I$-content $n_{I}(\mu)$ of any $\mu \in L^{\prime} \cap I^{\perp}$ as follows: If $Q(\mu)=0$ we put 
$n_{I}(\mu)=0$. If $Q(\mu) \neq 0$ we let $n_{I}(\mu)$ be the unique nonzero integer such that

$$
\left(\mu, L \cap I^{\perp}\right)=n_{I}(\mu) \cdot \mathbb{Z}
$$

and $\operatorname{sgn}\left(n_{I}(\mu)\right) \cdot \mu \sim I$.

Now, if $d \in-2 \operatorname{disc}(L)\left(\mathbb{Q}^{\times}\right)^{2}$ and $\lambda \in L_{d}^{\prime}$, we let $I \in \operatorname{Iso}(L)$ be the isotropic line associated to $\lambda$ and let $\ell^{\prime} \in L^{\prime}$ be as in (3-2) such that $\left(\ell^{\prime}, I\right)=\mathbb{Z}$. We choose a function $h_{\lambda} \in k(X)^{\times}$such that

$$
h_{\lambda}= \begin{cases}1-e^{2 \pi i\left(\lambda, \ell^{\prime}\right)} q_{I}^{n_{I}(\lambda)}+O\left(q_{I}^{m_{I}}\right) & \text { at the cusp } c_{I}, \\ 1+O\left(q_{J}^{m_{J}}\right), & \text { at all other cusps } c_{J} .\end{cases}
$$

The existence of $h_{\lambda}$ follows for instance from the approximation theorem for valuations, see page 29 in [Serre 1988]. If $\left(\lambda, \ell^{\prime}\right) \in \mathbb{Z}$, then $h_{\lambda}$ agrees with the function $h_{c_{I}, n_{I}(\lambda)} \in k(X)^{\times}$defined in (2-4). For $\varphi \in S_{L}$ we define

$$
[Z(d, \varphi)]_{\mathfrak{m}}=\sum_{\lambda \in L_{d}^{\prime} / \Gamma} \frac{1}{2 n_{I}(\lambda)}(\varphi(\lambda)+\varphi(-\lambda)) \cdot\left[\operatorname{div}\left(h_{\lambda}^{-1}\right)\right]_{\mathfrak{m}} .
$$

It is easily checked that this class is independent of the choices of the functions $h_{\lambda}$. If $d<0$ and $d \notin-2 \operatorname{disc}(L)\left(\mathbb{Q}^{\times}\right)^{2}$, we put $[Z(d, \varphi)]_{\mathfrak{m}}=0$.

Finally, for all $d \in \mathbb{Q}$ we write $[Z(d)]_{\mathfrak{m}}$ for the element of

$$
\operatorname{Hom}\left(S_{L}, J_{\mathfrak{m}}(X)_{\mathbb{C}}\right) \cong J_{\mathfrak{m}}(X)_{\mathbb{C}} \otimes S_{L}^{\vee}
$$

given by $\varphi \mapsto[Z(d, \varphi)]_{\mathfrak{m}}$.

The classes $[Z(d, \varphi)]_{\mathfrak{m}}$ with $d<0$ can also be expressed in a slightly different way. To this end, for an isotropic line $I$ we define

$$
L_{d, I}^{\prime}=\left\{\lambda \in L_{d}^{\prime}: \lambda \perp I \text { and } \lambda \sim I\right\} .
$$

Lemma 4.1. For $d<0$ we have

$$
[Z(d, \varphi)]_{\mathfrak{m}}=\sum_{I \in \operatorname{Iso}(L) / \Gamma} \sum_{\lambda \in L_{d, I}^{\prime} / I} \frac{1}{2}(\varphi(\lambda)+\varphi(-\lambda)) \cdot\left[\operatorname{div}\left(h_{\lambda}^{-1}\right)\right]_{\mathfrak{m}} .
$$

Proof. If $L_{d, I}$ is nonempty and if we fix $\lambda_{0} \in L_{d, I}^{\prime}$, we have

$$
\begin{aligned}
L_{d, I}^{\prime} & =\left\{\lambda_{0}+a \ell / N_{I}: a \in \mathbb{Z}\right\}, \\
L_{d, I}^{\prime} / \Gamma_{I} & =\left\{\lambda_{0}+a \ell / N_{I}: a \in \mathbb{Z} / N_{I} n_{I}\left(\lambda_{0}\right) \mathbb{Z}\right\}, \\
L_{d, I}^{\prime} / I & =\left\{\lambda_{0}+a \ell / N_{I}: a \in \mathbb{Z} / N_{I} \mathbb{Z}\right\} .
\end{aligned}
$$

This implies the assertion. 
An abstract modularity theorem. To describe the relations in the generalized Jacobian among the classes $[Z(d)]_{\mathfrak{m}}$ we form the generating series

$$
A_{\mathfrak{m}}(\tau)=\sum_{d \in \frac{1}{N} \mathbb{Z}}[Z(d)]_{\mathfrak{m}} \cdot q^{d} \in S_{L}^{\vee}((q)) \otimes J_{\mathfrak{m}}^{\text {add }}(X)_{\mathbb{C}} .
$$

It is a formal Laurent series in the variable ${ }^{1} q=e^{2 \pi i \tau}$, where $\tau \in \mathbb{H}$, with exponents in $\frac{1}{N} \mathbb{Z}$ and coefficients in $S_{L}^{\vee} \otimes J_{\mathfrak{m}}^{\text {add }}(X)_{\mathbb{C}}$.

Theorem 4.2. The generating series $A_{\mathfrak{m}}(\tau)$ is the q-expansion of a weakly holomorphic modular form in $M_{3 / 2}^{!}\left(\omega_{L}^{\vee}\right) \otimes J_{\mathfrak{m}}^{\text {add }}(X)_{\mathbb{C}}$.

To prove this result, we use the following variant of Borcherds' modularity criterion [Borcherds 1999, Theorem 3.1]. Let $\rho$ be a finite dimensional representation of $\mathrm{Mp}_{2}(\mathbb{Z})$ on a complex vector space $V$ which is trivial on some congruence subgroup. The stabilizer in $\mathrm{Mp}_{2}(\mathbb{Z})$ of the cusp $\infty$ is generated by the elements $T=\left(\left(\begin{array}{ll}1 & 1 \\ 0 & 1\end{array}\right), 1\right)$ and $Z=\left(\left(\begin{array}{cc}-1 & 0 \\ 0 & -1\end{array}\right), i\right)$. The hypothesis on $\rho$ implies that some power of $\rho(T)$ is the identity, and therefore all eigenvalues of $\rho(T)$ are roots of unity. If $g \in M_{k}^{!}(\rho)$ is a weakly holomorphic modular form for $\mathrm{Mp}_{2}(\mathbb{Z})$ of weight $k \in \frac{1}{2} \mathbb{Z}$ with representation $\rho$, then it has a Fourier expansion

$$
g(\tau)=\sum_{n \in \mathbb{Q}} a(n) \cdot q^{n},
$$

where the coefficients $a(n) \in V$ satisfy the conditions

$$
\begin{aligned}
& \rho(T) a(n)=e^{2 \pi i n} a(n), \\
& \rho(Z) a(n)=e^{-\pi i k} a(n) .
\end{aligned}
$$

We write $\rho^{\vee}$ for the representation dual to $\rho$, and denote by $(\cdot, \cdot)$ the natural pairing $V \times V^{\vee} \rightarrow \mathbb{C}$.

Proposition 4.3. A formal Laurent series

$$
g(\tau)=\sum_{n \in \mathbb{Q}} a(n) \cdot q^{n} \in V((q)),
$$

with coefficients a $(n)$ satisfying the conditions (4-10) and (4-11) is the q-expansion of a weakly holomorphic modular form in $M_{k}^{!}(\rho)$ if and only if

$$
\sum_{n \in \mathbb{Q}}(a(n), c(-n))=0
$$

for all

$$
f(\tau)=\sum_{n \in \mathbb{Q}} c(n) \cdot q^{n} \in M_{2-k}^{!}\left(\rho^{\vee}\right) .
$$

${ }^{1}$ Confusion with the local parameter $q_{I}$ at the $\operatorname{cusp} c_{I}$ of $X$ should not be possible. 
Proof. This result is proved in Section 3 of [Borcherds 1999] in the special case when $g$ is actually a formal power series. The same proof applies to our slightly more general case, if we replace the vector bundle of modular forms of type $\rho$ by a twist with a power of the line bundle $\mathcal{L}(\infty)$ corresponding to the cusp at $\infty$.

Alternatively, we may replace the $q$-series $g$ by the $q$-series $g^{\prime}=\Delta^{j} g$ for a positive integer $j$ such that $\Delta^{j} g$ is a power series. Here $\Delta$ is the normalized cusp form of weight 12. Then one can literally apply [Borcherds 1999, Theorem 3.1] to $g^{\prime}$ to deduce modularity in $M_{k+12 j}(\rho)$ of this power series. Dividing out the power of $\Delta$ again, we obtain the result.

Proof of Theorem 4.2. According to Proposition 4.3 with $\rho=\omega_{L}^{\vee}$, it suffices to show that

$$
\sum_{d \in \mathbb{Q}}\left(c(-d),[Z(d)]_{\mathfrak{m}}\right)=0 \in J_{\mathfrak{m}}^{\mathrm{add}}(X)_{\mathbb{C}}
$$

for every

$$
f(\tau)=\sum_{d \in \mathbb{Q}} c(d) \cdot q^{d} \in M_{1 / 2}^{!}\left(\omega_{L}\right)
$$

Since the space $M_{1 / 2}^{!}\left(\omega_{L}\right)$ has a basis of modular forms with integral coefficients [McGraw 2003], it suffices to check that for every $f$ with integral coefficients the relation (4-12) holds. For $\mu \in L^{\prime}$ we put $c(d, \mu)=c(d)(\mu)$.

Let $\Psi(z, f)$ be the Borcherds lift of $f$ as in [Borcherds 1998, Theorem 13.3]. This is a meromorphic modular form on $\mathcal{D}^{+}$for the group $\Gamma$ of weight $c(0,0)$ with some multiplier system of finite order. Its divisor on $X$ is given by

$$
\operatorname{div}(\Psi(z, f))=\sum_{d>0}(c(-d), Z(d))+B(f)
$$

where $B(f)$ is a divisor of degree $\frac{1}{12} c(0,0)$ supported at the cusps of $X$. Let $I \in \operatorname{Iso}(L)$. To determine the behavior of $\Psi(z, f)$ near the cusp $c_{I}$, we identify $\mathcal{D}^{+}$ with the upper complex half-plane $\mathbb{U}$ using (3-4). Then $\Psi(w, f)$ has the infinite product expansion

$$
\Psi(w, f)=R_{I} \cdot q_{I}^{\rho_{I}} \prod_{\substack{\lambda \in\left(L^{\prime} \cap I^{\perp}\right) / I \\ n_{I}(\lambda)>0}}\left(1-e^{2 \pi i\left(\lambda, \ell^{\prime}\right)} q_{I}^{n_{I}(\lambda)}\right)^{c(-Q(\lambda), \lambda)},
$$

which converges near the cusp $c_{I}$, that is, for $w \in \mathbb{W}$ with sufficiently large imaginary part. Here the product runs over vectors $\lambda$ of negative norm which are associated to $I$, and $\rho_{I} \in \mathbb{Q}$ is the Weyl vector at the $\operatorname{cusp} c_{I}$ corresponding to $f$. Moreover, the quantity $R_{I}$ is some constant in $k^{\times}$of modulus 1 times

$$
\prod_{\substack{a \in \mathbb{Z} / N_{I} \mathbb{Z} \\ a \neq 0}}\left(1-e^{2 \pi i a / N_{I}}\right)^{c\left(0, a \ell / N_{I}\right) / 2} .
$$


Hence, the (finite) product

$$
\Psi(w, f) \times R_{I}^{-1} \prod_{\substack{\lambda \in\left(L^{\prime} \cap I^{\perp}\right) / I \\ m_{I}>n_{I}(\lambda)>0}} h_{\lambda}^{-c(-Q(\lambda), \lambda)}
$$

is a meromorphic modular form of weight $c(0,0)$ satisfying the condition (2-6) at $c_{I}$. There exists a degree-zero divisor $D$ supported on $S$ such that the finite product

$$
\Psi(w, f) \times u_{D,\left(R_{I}\right)}^{-1} \times \prod_{I \in \operatorname{Iso}(L) / \Gamma} \prod_{\substack{\lambda \in\left(L^{\prime} \cap I^{\perp}\right) / I \\ m_{I}>n_{I}(\lambda)>0}} h_{\lambda}^{-c(-Q(\lambda), \lambda)}
$$

is a meromorphic modular form of weight $c(0,0)$ satisfying the condition $(2-6)$ at all cusps and having order 0 at all cusps different from $c_{I_{0}}$. Here $u_{D, r} \in H_{\mathbb{G}_{m}, \mathfrak{m}}$ denotes the function defined in (4-1).

By the choice of the base point $s_{0}=c_{I_{0}}$ in (2-7), the class of the line bundle $\mathcal{M}_{c(0,0)}$ in $J_{\mathfrak{m}}(X)$ is given by

$$
\begin{aligned}
{\left[\mathcal{M}_{c(0,0)}\right]_{\mathfrak{m}}=[\operatorname{div}(\Psi(f))} & \left.-\operatorname{deg}\left(\mathcal{M}_{c(0,0)}\right)\left(c_{I_{0}}\right)\right]_{\mathfrak{m}}-\left[\operatorname{div}\left(u_{D,\left(R_{I}\right)}\right)\right]_{\mathfrak{m}} \\
- & \sum_{I \in \operatorname{Iso}(L) / \Gamma} \sum_{\substack{\lambda \in\left(L^{\prime} \cap I^{\perp}\right) / I \\
m_{I}>n_{I}(\lambda)>0}} c(-Q(\lambda), \lambda) \cdot\left[\operatorname{div}\left(h_{\lambda}\right)\right]_{\mathfrak{m}} .
\end{aligned}
$$

Using Lemma 4.1, we see that

$$
\sum_{I \in \operatorname{Iso}(L) / \Gamma} \sum_{\substack{\lambda \in\left(L^{\prime} \cap I^{\perp}\right) / I \\ m_{I}>n_{I}(\lambda)>0}} c(-Q(\lambda), \lambda) \cdot\left[\operatorname{div}\left(h_{\lambda}\right)\right]_{\mathfrak{m}}=-\sum_{d<0}\left(c(-d),[Z(d)]_{\mathfrak{m}}\right) .
$$

Inserting this into (4-15), we obtain the relation

$-c(0,0)\left[\mathcal{M}_{-1}\right]_{\mathfrak{m}}=\sum_{d>0}\left(c(-d),[Z(d)]_{\mathfrak{m}}\right)+\sum_{d<0}\left(c(-d),[Z(d)]_{\mathfrak{m}}\right)-\left[\operatorname{div}\left(u_{D,\left(R_{I}\right)}\right)\right]_{\mathfrak{m}}$ in $J_{\mathfrak{m}}(X)_{\mathbb{C}}$. This implies (4-12) in $J_{\mathfrak{m}}^{\text {add }}(X)_{\mathbb{C}}$, concluding the proof.

Remark 4.4. To be able to describe the generating series in $J_{\mathfrak{m}}(X)_{\mathbb{C}}$ instead of in the quotient $J_{\mathfrak{m}}^{\text {add }}(X)_{\mathbb{C}}$, we would have to know the normalizing factors $R_{I}$ in (4-14) more precisely. It would be very interesting to understand these better. Are they roots of unity?

By the Manin-Drinfed theorem, the natural homomorphism $J_{\mathfrak{m}}(X) \rightarrow J(X)$ induces a linear map

$$
J_{\mathfrak{m}}^{\mathrm{add}}(X)_{\mathbb{C}} \rightarrow J(X)_{\mathbb{C}}
$$

The classes $[Z(d)]_{\mathfrak{m}}$ with $d \leq 0$ are in the kernel. Applying this map coefficientwise to the generating series $A_{\mathfrak{m}}$ in Theorem 4.2, we obtain the Gross-Kohnen-Zagier 
theorem.

Corollary 4.5 (Gross-Kohnen-Zagier). The generating series

$$
A_{0}(\tau)=\sum_{d>0}[Z(d)]_{0} \cdot q^{d}
$$

of the classes of the Heegner divisors in the Jacobian $J(X)_{\mathbb{C}}$ is the q-expansion of a cusp form in $S_{3 / 2}\left(\omega_{L}^{\vee}\right) \otimes J(X)_{\mathbb{C}}$.

\section{Traces of singular moduli}

Here we show that every harmonic Maass form of weight zero with vanishing constant terms defines a linear functional of the generalized Jacobian $J_{\mathfrak{m}}^{\text {add }}(X)_{\mathbb{C}}$. Applying it to the generating series $A_{\mathfrak{m}}$, one obtains modularity results for traces of $\mathrm{CM}$ values of harmonic Maass forms and weakly holomorphic modular forms as in [Zagier 2002; Bruinier and Funke 2006].

Let $H_{k}^{+}(\Gamma)$ be the space of harmonic Maass forms of weight $k$ for $\Gamma$ as in [Bruinier and Funke 2004, Section 3]. Recall that there is a surjective differential operator $\xi_{k}: H_{k}^{+}(\Gamma) \rightarrow S_{2-k}(\Gamma)$ to cusp forms of "dual" weight $2-k$.

For the rest of this section we fix a nonzero $F \in H_{0}^{+}(\Gamma)$. We denote the holomorphic part of the Fourier expansion of $F$ at the cusp $c_{I}$ corresponding to $I \in \operatorname{Iso}(L)$ by

$$
F_{I}^{+}=\sum_{j \in \mathbb{Z}} c_{F, I}^{+}(j) \cdot q_{I}^{j} .
$$

We define the order of $F$ at the $\operatorname{cusp} c_{I}$ by

$$
\operatorname{ord}_{c_{I}}(F)=\min \left\{j \in \mathbb{Z}: c_{F, I}^{+}(j) \neq 0\right\} .
$$

Proposition 5.1. Assume that for all $I \in \operatorname{Iso}(L)$ we have $\operatorname{ord}_{c_{I}}(F)>-m_{I}$ and $c_{F, I}^{+}(0)=0$.

(i) There is a linear map,

$$
\operatorname{tr}_{F}: J_{\mathfrak{m}}(X) \rightarrow \mathbb{C}
$$

defined by

$$
[D]_{\mathfrak{m}} \mapsto \operatorname{tr}_{F}(D):=\sum_{a \in \operatorname{supp}(D) \backslash S} n_{a} \cdot F(a),
$$

for divisors $D=\sum_{a} n_{a} \cdot(a)$ in $\operatorname{Div}^{0}(X)$.

(ii) The map $\operatorname{tr}_{F}$ vanishes on $H_{\mathbb{G}_{m}}$, m and factors through $J_{\mathfrak{m}}^{\operatorname{add}}(X)$.

Proof. (i) We have to show that $\operatorname{tr}_{F}(D)=0$, for every divisor $D=\operatorname{div}(g) \in P_{\mathfrak{m}}(X)$ given by a rational function $g \in k(X)^{\times}$satisfying

$$
q_{I}^{-\operatorname{ord}_{c_{I}}(g)} g=1+O\left(q_{I}^{m_{I}}\right)
$$


at every cusp $c_{I}$. The expansion of the logarithmic derivative of $g$ with respect to the local parameter $q_{I}$ at $c_{I}$ is of the form

$$
\frac{d g}{g}=\operatorname{ord}_{c_{I}}(g) q_{I}^{-1}+O\left(q_{I}^{m_{I}-1}\right)
$$

If $F$ is weakly holomorphic, then $\eta:=F(d g / g)$ is a meromorphic 1-form on $X$. Hence, by the residue theorem, the sum of the residues of $\eta$ vanishes, and we have

$$
\sum_{a \in X \backslash S} \operatorname{res}_{a}(\eta)=-\sum_{a \in S} \operatorname{res}_{a}(\eta) .
$$

The left-hand side of this equality is given by $\operatorname{tr}_{F}(D)$, while the right-hand side satisfies

$$
\begin{aligned}
\sum_{a \in S} \operatorname{res}_{a}(\eta) & =\sum_{I \in \operatorname{Iso}(L) / \Gamma} \operatorname{res}_{c_{I}}(\eta) \\
& =\sum_{I \in \operatorname{Iso}(L) / \Gamma} \operatorname{res}_{q_{I}=0}\left(\left(\operatorname{ord}_{c_{I}}(g) q_{I}^{-1}+O\left(q_{I}^{m_{I}-1}\right)\right) \sum_{j>-m_{I}} c_{F, I}^{+}(j) \cdot q_{I}^{j}\right)=0 .
\end{aligned}
$$

Here we have also used the fact that $c_{F, I}^{+}(0)=0$ for all $I$.

To prove the assertion for general $F \in H_{0}^{+}(\Gamma)$, we let $X_{\varepsilon}$ be the manifold with boundary obtained from $X$ by cutting out small oriented discs of radius $\varepsilon$ around the points in $\operatorname{supp}(\operatorname{div}(g)) \cup S$. Then for the 1 -form $\eta:=F(d g / g)$ it is still true that

$$
\lim _{\varepsilon \rightarrow 0} \int_{\partial X_{\varepsilon}} \eta=0 \text {. }
$$

Indeed, we have

$$
\int_{\partial X_{\varepsilon}} \eta=\int_{\partial X_{\varepsilon}} F \cdot \partial \log |g|^{2}=\int_{X_{\varepsilon}} d\left(F \cdot \partial \log |g|^{2}\right) .
$$

Since $\log |g|^{2}$ and $F$ are harmonic functions on $X_{\varepsilon}$, we find that

$$
\int_{\partial X_{\varepsilon}} \eta=\int_{X_{\varepsilon}}(\bar{\partial} F) \wedge\left(\partial \log |g|^{2}\right)=-\int_{X_{\varepsilon}} \partial\left((\bar{\partial} F) \log |g|^{2}\right)=-\int_{\partial X_{\varepsilon}}(\bar{\partial} F) \log |g|^{2} .
$$

In the latter integral, the differential $\bar{\partial} F=\overline{\xi_{0}(F)} d \bar{z}$ is antiholomorphic (hence smooth) on all of $X$. Since $\log |g|^{2}$ has only logarithmic singularities, the integral vanishes in the limit $\varepsilon \rightarrow 0$.

On the other hand, a local computation shows that

$$
\lim _{\varepsilon \rightarrow 0} \int_{\partial X_{\varepsilon}} \eta=\operatorname{tr}_{F}(D)+\sum_{I \in \operatorname{Iso}(L) / \Gamma} \operatorname{res}_{c_{I}}\left(F_{I}^{+} \cdot \frac{d g}{g}\right) .
$$

The vanishing of the second summand on the right-hand side follows as before, proving that $\operatorname{tr}_{F}(D)=0$ again. 
(ii) Let $u_{D, r}$ be as in (4-1). The same argument shows that $\operatorname{tr}_{F}\left(\operatorname{div}\left(u_{D, r}\right)\right)$ vanishes and $\operatorname{tr}_{F}$ factors through $J_{\mathfrak{m}}^{\text {add }}(X)$.

Theorem 5.2. Assume that $\operatorname{ord}_{c_{I}}(F)>-m_{I}$ and $c_{F, I}^{+}(0)=0$ for all $I \in \operatorname{Iso}(L)$. Then $\operatorname{tr}_{F}\left(A_{\mathfrak{m}}\right)$ is a weakly holomorphic modular form in $M_{3 / 2}^{!}\left(\omega_{L}^{\vee}\right)$, and $\operatorname{tr}_{F}\left(A_{\mathfrak{m}}\right)(\varphi)=\sum_{d<0} \operatorname{tr}_{F}\left([Z(d, \varphi)]_{\mathfrak{m}}\right) \cdot q^{d}+\operatorname{tr}_{F}\left(\left[\mathcal{M}_{-1}\right]_{\mathfrak{m}}\right) \varphi(0)+\sum_{d>0} F(Y(d, \varphi)) \cdot q^{d}$ Moreover, for $d<0$ the quantity $\operatorname{tr}_{F}\left([Z(d, \varphi)]_{\mathfrak{m}}\right)$ is given by the finite sum $\operatorname{tr}_{F}\left([Z(d, \varphi)]_{\mathfrak{m}}\right)$

$$
=-\frac{1}{2} \sum_{I \in \operatorname{Iso}(L) / \Gamma} \sum_{\lambda \in L_{d, I}^{\prime} / \Gamma_{I}}(\varphi(\lambda)+\varphi(-\lambda)) \cdot \sum_{j \geq 1} e^{2 \pi i\left(\lambda, \ell_{I}^{\prime}\right) j} c_{F, I}^{+}\left(-n_{I}(\lambda) j\right) .
$$

Proof. The modularity of $\operatorname{tr}_{F}\left(A_{\mathfrak{m}}\right)$ is a direct consequence of Theorem 4.2 and Proposition 5.1 .

We now compute the $q$-expansion. For $d>0$ and $\varphi \in S_{L}$ we have by definition of the $\operatorname{map} \operatorname{tr}_{F}$ that

$$
\operatorname{tr}_{F}\left([Z(d, \varphi)]_{\mathfrak{m}}\right)=F(Y(d, \varphi))
$$

If $d<0$ and $d \in-2 \operatorname{disc}(L)\left(\mathbb{Q}^{\times}\right)^{2}$, we obtain by the definition of the class $[Z(d)]_{\mathfrak{m}}$ that

$$
\begin{aligned}
\operatorname{tr}_{F}\left([Z(d, \varphi)]_{\mathfrak{m}}\right) & =\sum_{\lambda \in L_{d}^{\prime} / \Gamma} \frac{1}{2 n_{I}(\lambda)}(\varphi(\lambda)+\varphi(-\lambda)) \cdot \operatorname{tr}_{F}\left(\operatorname{div}\left(h_{\lambda}^{-1}\right)\right) \\
& =-\sum_{I \in \operatorname{Iso}(L) / \Gamma} \sum_{\lambda \in L_{d, I}^{\prime} / \Gamma_{I}} \frac{1}{2 n_{I}(\lambda)}(\varphi(\lambda)+\varphi(-\lambda)) \cdot F\left(\operatorname{div}\left(h_{\lambda}\right)\right) .
\end{aligned}
$$

Arguing as in the proof of Proposition 5.1, in particular (5-2), we find for $\lambda \in L_{d, I}^{\prime}$ that

$$
\begin{aligned}
F\left(\operatorname{div}\left(h_{\lambda}\right)\right) & =-\sum_{J \in \operatorname{Iso}(L) / \Gamma} \operatorname{res}_{c_{J}}\left(F_{J}^{+} \cdot \frac{d h_{\lambda}}{h_{\lambda}}\right) \\
& =\operatorname{res}_{c_{I}}\left(F_{I}^{+} \cdot \frac{n_{I}(\lambda) \cdot e^{2 \pi i\left(\lambda, \ell_{I}^{\prime}\right)} q_{I}^{n_{I}(\lambda)-1}+O\left(q_{I}^{m_{I}-1}\right)}{1-e^{2 \pi i\left(\lambda, \ell_{I}^{\prime}\right)} q_{I}^{n_{I}(\lambda)}}\right) \\
& =n_{I}(\lambda) \sum_{j \geq 1} e^{2 \pi i\left(\lambda, \ell_{I}^{\prime}\right) j} c_{F, I}^{+}\left(-n_{I}(\lambda) j\right) .
\end{aligned}
$$

Inserting this into the previous equation, we obtain the assertion.

Remark 5.3. The constant term $\operatorname{tr}_{F}\left([Z(0, \varphi)]_{\mathfrak{m}}\right)$ can also be computed explicitly, see Proposition 5.4 for an example. 
An example. Consider the modular curve $X_{0}(M)$ for a squarefree $M \in \mathbb{Z}_{>0}$. Let $L$ be the lattice

$$
L=\left\{\left(\begin{array}{cc}
b & a / M \\
c & -b
\end{array}\right): a, b, c \in \mathbb{Z}\right\},
$$

with the quadratic form $Q(X)=M \operatorname{det}(X)$. Then $L^{\prime} / L \cong \mathbb{Z} / 2 M \mathbb{Z}$ and $\mathrm{SO}^{+}(L)$ is isomorphic to the extension $\Gamma_{0}^{*}(M)$ of $\Gamma_{0}(M)$ by the Atkin-Lehner involutions. The discriminant kernel subgroup $\Gamma$ is isomorphic to $\Gamma_{0}(M)$, and the modular curve $X_{\Gamma}$ is isomorphic to $X_{0}(M)$ with the cusp associated to the isotropic line $I_{0}=\mathbb{Z}\left(\begin{array}{ll}0 & 0 \\ 1 & 0\end{array}\right)$ corresponding to $\infty$; see, e.g., [Bruinier and Ono 2010, Section 2.4].

The group $\Gamma_{0}^{*}(M)$ acts transitively on $\operatorname{Iso}(L)$, and the orbits are represented by the lines $I_{D}=W_{D} \cdot I_{0}$ for the positive divisors $D \mid M$. Here $W_{D} \in \mathrm{PGL}_{2}^{+}(\mathbb{Q})$ denotes the Atkin-Lehner involution with index $D$. In particular, the set $S$ of cusps of $X_{0}(M)$ is in bijection with the set of positive divisors of $M$. If $I \in \operatorname{Iso}(L)$, we write $D_{I}$ for the unique positive divisor of $M$ such that $I$ is equivalent to $W_{D_{I}} \cdot I_{0}$ under $\Gamma$. Let $F \in H_{0}^{+}(\Gamma)$ be a harmonic Maass form. The expansion of $F$ at the cusp $I_{D}$ as in (5-1) is given by the Fourier expansion of $F \mid W_{D}$.

Proposition 5.4. Assume that for all $I \in \operatorname{Iso}(L)$ we have $\operatorname{ord}_{c_{I}}(F)>-m_{I}$ and $c_{F, I}^{+}(0)=0$. The constant term of the generating series $\operatorname{tr}_{F}\left(A_{\mathfrak{m}}\right)$ is given by

$$
\operatorname{tr}_{F}\left(\left[\mathcal{M}_{-1}\right]_{\mathfrak{m}}\right)=2 \sum_{D \mid M} \sum_{j \geq 1} c_{F, I_{D}}^{+}(-j) \cdot D \cdot \sigma_{1}(j / D) .
$$

Remark 5.5. As shown in [Bruinier and Funke 2006, Remark 4.9], the right-hand side above is also equal to $-\frac{1}{4 \pi} \int_{\Gamma_{0}(M) \backslash \mathbb{M}}^{\mathrm{reg}} F d \mu$. The proposition gives a geometric interpretation of this regularized integral.

Proof of Proposition 5.4. We use the notation of the proof of Theorem 5.2. By linearity it suffices to compute the class of the line bundle $\mathcal{M}_{12}$. Since $X_{\Gamma} \cong X_{0}(M)$, a section of this line bundle is the usual discriminant function given by

$$
\Delta=q \prod_{n \geq 1}\left(1-q^{n}\right)^{24} .
$$

To compute the class of $\mathcal{M}_{12}$ in the generalized Jacobian, we have to modify this section by multiplying with rational functions such that the local conditions (2-6) at the cusps are satisfied. It is easily checked that

$$
\Delta \mid W_{D}=D^{-6} \Delta(D \tau)=D^{-6} q^{D} \prod_{n \geq 1}\left(1-q^{D n}\right)^{24} .
$$

This implies that the section

$$
s=\Delta \cdot \prod_{I \in \operatorname{Iso}(L) / \Gamma} \prod_{\substack{\lambda \in\left(L^{\prime} \cap I^{\perp}\right) / I \\ m_{I}>n_{I}(\lambda)>0}} h_{D_{I} \lambda}^{-24}
$$


has the expansion

$$
s=D^{-6} q_{I_{D}}^{D} \cdot\left(1+O\left(q_{I_{D}}^{D m_{I}}\right)\right)
$$

at the cusp $I_{D}$. For the $|S|$-tuple $r=\left(D^{6}\right)_{D \mid M}$, and the function $u_{0, r} \in \mathbb{Q}\left(X_{0}(M)\right)^{\times}$, the section $s \cdot u_{0, r}$ of $\mathcal{M}_{12}$ satisfies the local conditions (2-6) at all cusps. Therefore, in view of (2-7) and Proposition 5.1 (ii), we have

$$
\begin{aligned}
\operatorname{tr}_{F}\left(\left[\mathcal{M}_{12}\right]_{\mathfrak{m}}\right) & =\operatorname{tr}_{F}\left(\left[\operatorname{div}\left(s \cdot u_{0, r}\right)-\operatorname{deg}\left(\mathcal{M}_{12}\right) \cdot\left(c_{I_{0}}\right)\right]_{\mathfrak{m}}\right) \\
& =-24 \sum_{I \in \operatorname{Iso}(L) / \Gamma} \sum_{\substack{\lambda \in\left(L^{\prime} \cap I^{\perp}\right) / I \\
m_{I}>n_{I}(\lambda)>0}} F\left(\operatorname{div}\left(h_{D_{I} \lambda}\right)\right) .
\end{aligned}
$$

Using formula (5-3), we get

$$
\begin{aligned}
\operatorname{tr}_{F}\left(\left[\mathcal{M}_{12}\right]_{\mathfrak{m}}\right) & =-24 \sum_{I \in \operatorname{Iso}(L) / \Gamma} \sum_{n=1}^{m_{I}-1} D_{I} n \sum_{j \geq 1} c_{F, I}^{+}\left(-D_{I} n j\right) \\
& =-24 \sum_{D \mid M} \sum_{j \geq 1} c_{F, I_{D}}^{+}(-j) \cdot D \cdot \sigma_{1}(j / D) .
\end{aligned}
$$

This concludes the proof of the proposition.

We now explain how to obtain a scalar-valued generating series from $\operatorname{tr}_{F}\left(A_{\mathfrak{m}}\right)$. By means of the canonical pairing $\left(S_{L}, S_{L}^{\vee}\right) \rightarrow \mathbb{C}$, we define a map

$$
S_{L}^{\vee} \rightarrow \mathbb{C}, \quad u \mapsto\left(\chi_{1}, u\right),
$$

given by the pairing with the constant function $\chi_{1}$ with value 1 . It induces a map from $S_{L}^{\vee}$-valued to scalar-valued modular forms,

$$
M_{3 / 2}^{!}\left(\omega_{L}^{\vee}\right) \rightarrow M_{3 / 2}^{!}\left(\Gamma_{0}(4 M)\right), \quad f(\tau) \mapsto f^{\mathrm{scal}}(\tau):=f\left(\chi_{1}\right)(4 M \tau),
$$

see [Eichler and Zagier 1985, §5]. The image lies in the Kohnen plus-space. Applying this map to the generating series $A_{\mathfrak{m}}$ of Theorem 4.2, we obtain a scalar valued generating series which has level $4 M$. In particular, this implies Theorem 1.1 of the introduction. If we apply this map to Theorem 5.2 and use Proposition 5.4, we obtain:

Theorem 5.6. Let $L$ be as in (5-4). Assume that for all $I \in \operatorname{Iso}(L)$ we have $\operatorname{ord}_{c_{I}}(F)>-m_{I}$ and $c_{F, I}^{+}(0)=0$. Then $\operatorname{tr}_{F}\left(A_{\mathfrak{m}}^{\text {scal }}\right) \in M_{3 / 2}^{!}\left(\Gamma_{0}(4 M)\right)$, and

$$
\begin{aligned}
\operatorname{tr}_{F}\left(A_{\mathfrak{m}}^{\mathrm{scal}}\right)=- & \sum_{D \mid M} \sum_{b \geq 1} \sum_{n \geq 1} c_{F, I_{D}}^{+}(-b n) \cdot b \cdot q^{-b^{2}} \\
& +2 \sum_{D \mid M} \sum_{n \geq 1} c_{F, I_{D}}^{+}(-n) \cdot D \cdot \sigma_{1}(n / D)+\sum_{d \in \mathbb{Z}_{>0}} F\left(Y\left(d / 4 M, \chi_{1}\right)\right) \cdot q^{d} .
\end{aligned}
$$


When $M=p$ is a prime and $F$ is invariant under the Fricke involution, we obtain Theorem 1.4 of the introduction.

Now let $M=1$ and let $j=E_{4}^{3} / \Delta$ be the classical $j$-function. Write $J=j-744=$ $q^{-1}+196884 q+\cdots$ for the normalized Hauptmodul for $\mathrm{PSL}_{2}(\mathbb{Z})$ with vanishing constant term. Applying Theorem 5.6 with $F=J$, we recover Zagier's original result [2002]:

Corollary 5.7. The generating series

$$
-q^{-1}+2+\sum_{d \in \mathbb{Z}_{d>0}} J\left(Y\left(d / 4, \chi_{1}\right)\right) \cdot q^{d}
$$

of the traces of singular moduli is a weakly holomorphic modular form for $\Gamma_{0}(4)$ of weight $\frac{3}{2}$ in the plus-space.

\section{Generalizations}

In the section we describe some variants of our main results and indicate possible generalizations.

Modularity in the generalized class group. In the definition of the Heegner divisors $Z(d)$ we have projected to degree- 0 divisors by subtracting a suitable multiple of $\left(c_{I_{0}}\right)$. We now briefly describe what happens if we do not apply this projection and consider the divisors $Y(d, \varphi)$ defined in (3-7) for $d>0$. Then the corresponding generating series is a nonholomorphic modular form, where the nonholomorphic part is coming from a generalization of Zagier's weight- $\frac{3}{2}$ Eisenstein series.

We let $\mathrm{Cl}_{\mathfrak{m}}(X)$ be the generalized class group of $X$ with respect to the modulus $\mathfrak{m}$, which we define as the quotient of the group of divisors on $X$ defined over $k$ modulo the subgroup $P_{\mathfrak{m}}(X)$. Moreover, in analogy with (4-2) we put

$$
\mathrm{Cl}_{\mathfrak{m}}^{\mathrm{add}}(X)=\mathrm{Cl}_{\mathfrak{m}}(X) / H_{\mathbb{G}_{m}, \mathfrak{m}}
$$

If $d>0$, we write $[Y(d, \varphi)]_{\mathfrak{m}}$ for the class of the divisor $Y(d, \varphi)$ in $\mathrm{Cl}_{\mathfrak{m}}(X)$. For $d=0$ we put $[Y(0, \varphi)]_{\mathfrak{m}}=\varphi(0)\left[\mathcal{M}_{-1}\right]_{\mathfrak{m}}$, where the class in $\mathrm{Cl}_{\mathfrak{m}}(X)$ of a line bundle $\mathcal{L}$ is defined as in (2-7) but without the summand $\operatorname{deg}(\mathcal{L}) \cdot\left(s_{0}\right)$. Finally, for $d<0$ we let $[Y(d, \varphi)]_{\mathfrak{m}}=[Z(d, \varphi)]_{\mathfrak{m}}$.

Recall from [Funke 2002, Theorem 3.5] that there is a (nonholomorphic) weight$\frac{3}{2}$ Eisenstein series $E_{3 / 2, L}(\tau)$ whose coefficients with nonnegative index are given by the degrees of the $Y(d, \varphi)$ (see also [Kudla 2003]). It is a harmonic Maass form of weight $\frac{3}{2}$ for the group $\mathrm{Mp}_{2}(\mathbb{Z})$ with representation $\omega_{L}^{\vee}$ and generalizes Zagier's nonholomorphic Eisenstein series [1975]. Its Fourier expansion decomposes as

$$
E_{\frac{3}{2}, L}(\tau)=E_{\frac{3}{2}, L}^{+}(\tau)+E_{\frac{3}{2}, L}^{-}(\tau),
$$


where the holomorphic part is the generating series of the degrees of Heegner divisors,

$$
E_{\frac{3}{2}, L}^{+}(\tau)=\sum_{d \geq 0} \operatorname{deg}(Y(d)) \cdot q^{d},
$$

and the nonholomorphic part $E_{3 / 2, L}^{-}$is a period integral of a linear combination of unary theta series. We obtain the following variant of Theorem 4.2.

Theorem 6.1. The generating series

$$
\tilde{A}_{\mathfrak{m}}(\tau)=\sum_{d \in \frac{1}{N} \mathbb{Z}}[Y(d)]_{\mathfrak{m}} \cdot q^{d}+E_{\frac{3}{2}, L}^{-} \cdot\left(c_{I_{0}}\right)
$$

is a nonholomorphic modular form of weight $\frac{3}{2}$ for $\mathrm{Mp}_{2}(\mathbb{Z})$ with representation $\omega_{L}^{\vee}$ with values in $\mathrm{Cl}_{\mathfrak{m}}^{\text {add }}(X)$. Moreover, we have

$$
A_{\mathfrak{m}}=\tilde{A}_{\mathfrak{m}}-E_{\frac{3}{2}, L} \cdot\left(c_{I_{0}}\right) .
$$

Twists by genus characters. Let $L$ be the lattice of page 1294 for a squarefree $M \in \mathbb{Z}_{>0}$, and recall that $\Gamma \cong \Gamma_{0}(M)$. For a discriminant $\Delta \neq 1$ and $r \in \mathbb{Z}$ such that $\Delta \equiv r^{2} \bmod 4 M$, we can define a generalized genus character $\chi_{\Delta}$ on $L^{\prime}$ as in [Gross et al. 1987, Section I.2] and [Bruinier and Ono 2010, Section 4] let

$\chi_{\Delta}(\lambda)= \begin{cases}\left(\frac{\Delta}{n}\right) & \text { if } \Delta \mid b^{2}-4 M a c \text { and }\left(b^{2}-4 M a c\right) / \Delta \text { is a square modulo } 4 M \\ 0 & \text { otherwise, }\end{cases}$ with $\lambda=\left(\begin{array}{cc}b / 2 M & -a / M \\ c & -b / 2 M\end{array}\right) \in L^{\prime}$ and $n \in \mathbb{Z}$ any integer prime to $\Delta$ represented by one of the quadratic forms $\left[M_{1} a, b, M_{2} c\right]$ with $M_{1} M_{2}=M$. Note that $\chi_{\Delta}$ is invariant under $\mathrm{SO}^{+}(L)$.

If $\lambda \in L^{\prime}$ with $Q(\lambda) \in-4 M\left(\mathbb{Q}^{\times}\right)^{2}$, let $I$ be the isotropic line associated with $\lambda$, and let $h_{\Delta, \lambda} \in \mathbb{Q}(\sqrt{\Delta})(X)^{\times}$be a rational function with the following expansions:

$$
h_{\Delta, \lambda}= \begin{cases}\prod_{b \in \mathbb{Z} / \Delta \mathbb{Z}}\left(1-e^{2 \pi i b / \Delta} q_{I}^{n_{I}(\lambda)}\right)^{\left(\frac{\Delta}{b}\right)}+O\left(q_{I}^{m_{I}}\right) & \text { at the cusp } c_{I}, \\ 1+O\left(q_{J}^{m_{J}}\right) & \text { at all other cusps } c_{J} .\end{cases}
$$

Suppose that $(\Delta, 2 M)=1$, or equivalently $(r, 2 M)=1$. For each $d \in \frac{1}{4 M} \mathbb{Z}$ and $\varphi \in S_{L}$, we can define the divisor $Z_{\Delta, r}(d, \varphi) \in \operatorname{Div}^{0}(X)_{\mathbb{C}}$ by

$$
Z_{\Delta, r}(d, \varphi):= \begin{cases}\sum_{\lambda \in L_{d|\Delta|}^{\prime} / \Gamma} \frac{\chi_{\Delta}(\lambda) \varphi\left(r^{-1} \lambda\right)}{2\left|\Gamma_{\lambda}\right|} \cdot\left(z_{\lambda}\right) & \text { if } d>0, \\ \sum_{\lambda \in L_{d /|\Delta|}^{\prime} / \Gamma} \frac{\varphi(r \lambda)+\operatorname{sgn}(\Delta) \varphi(-r \lambda)}{2 n_{I}(\lambda)} \operatorname{div}\left(h_{\Delta, \lambda}^{-1}\right) & \text { if } d \in-\frac{|\Delta|}{4 M}\left(\mathbb{Z}_{>0}\right)^{2}, \\ 0 & \text { otherwise. }\end{cases}
$$


All these divisors are defined over $\mathbb{Q}(\sqrt{\Delta})$ and have coefficients in the field of definition of $\varphi$. We write $\left[Z_{\Delta, r}(d)\right]_{\mathfrak{m}} \in S_{L}^{\vee} \otimes J_{\mathfrak{m}}^{\text {add }}(X)$ for the element that sends $\varphi$ to $\left[Z_{\Delta, r}(d, \varphi)\right]_{\mathfrak{m}} \in J_{\mathfrak{m}}^{\text {add }}(X)$. Define the representation $\tilde{\omega}_{L}$ to be $\omega_{L}$ if $\Delta>0$ and $\bar{\omega}_{L}$ if $\Delta<0$. Then we have the following abstract modularity result.

Theorem 6.2. The generating series

$$
A_{\Delta, r, \mathfrak{m}}(\tau):=\sum_{d \in \frac{1}{4 M} \mathbb{Z}}\left[Z_{\Delta, r}(d)\right]_{\mathfrak{m}} \cdot q^{d} \in S_{L}^{\vee}((q)) \otimes J_{\mathfrak{m}}^{\mathrm{add}}(X)_{\mathbb{C}}
$$

is the $q$-expansion of a weakly holomorphic modular form in $M_{3 / 2}^{!}\left(\tilde{\omega}_{L}\right) \otimes J_{\mathfrak{m}}^{\mathrm{add}}(X)_{\mathbb{C}}$.

This result comes out of calculating the effect of the intertwining operator in [Alfes and Ehlen 2013, Section 3] applied to the generating series $A_{\mathfrak{m}}(\tau)$ associated to the scaled lattice $(\Delta L, Q(\cdot) /|\Delta|)$. The conditions that $M$ is squarefree and $(\Delta, 2 M)=1$ are imposed to simplify the definition of $Z_{\Delta, r}(d, \varphi)$ and can be removed with a more complicated definition of the classes. Note that it is necessary for $\operatorname{sgn}(\Delta)$, which determines the parity of $\tilde{\omega}_{L}$, to appear in the definition of $Z_{\Delta, r}(d, \varphi)$. Alternatively, one could use the twisted Borcherds products in [Bruinier and Ono 2010, Theorem 6.1] to give a proof of Theorem 6.2 along the same line as that of Theorem 4.2 above. By applying the functionals of Proposition 5.1 to the twisted generating series of Theorem 6.2, the main result of [Alfes and Ehlen 2013] on twisted traces of harmonic Maass forms can be recovered.

Other orthogonal Shimura varieties. The Gross-Kohnen-Zagier theorem has been generalized to higher dimensional orthogonal Shimura varieties in [Borcherds 1999]. Hence it is natural to ask whether our main results can also be generalized in the same direction. Let $L$ be an even lattice of signature $(n, 2)$, and let $\Gamma$ be the discriminant kernel subgroup of $\mathrm{SO}^{+}(L)$. Denote by $X_{\Gamma}$ a (suitable) toroidal compactification of the connected Shimura variety $Y_{\Gamma}$ associated to $\Gamma$. It would be interesting to define a generalized divisor class group as the group of divisors on $X_{\Gamma}$ modulo divisors of rational functions that satisfy certain growth conditions along the boundary of $X_{\Gamma}$. Is it possible to prove a modularity result analogous to Theorem 4.2 for the classes of special divisors? In this context, the product expansions obtained in [Kudla 2014] with respect to one-dimensional Baily-Borel boundary components may be helpful.

To illustrate this question, let us consider the easiest case for $n=2$ where the lattice $L$ is the even unimodular lattice of signature $(2,2)$. Then the variety $X_{\Gamma}$ can be identified with the product $X(1) \times X(1)$ of two copies of the compact modular curve of level 1. Special divisors on $X_{\Gamma}$ of positive index $d$ in the sense of [Kudla 1997] are given by the Hecke correspondences $Z(d)$. Let $q=\left(q_{1}, q_{2}\right)$ be the usual local coordinates near the boundary point $s=(\infty, \infty) \in X_{\Gamma}$. Let $m$ be a positive 
integer, and put $\mathfrak{m}=m \cdot(s)$. If $k=\left(k_{1}, k_{2}\right) \in \mathbb{Z}^{2}$ we briefly write $q^{k}=q_{1}^{k_{1}} q_{2}^{k_{2}}$, and for a meromorphic function $f$ in a neighborhood of $(\infty, \infty)$ we write $f=O\left(q^{m}\right)$ if in the Taylor expansion of $f$ at $(\infty, \infty)$ only terms of total degree at least $m$ occur.

Let $\operatorname{Div}_{\mathfrak{m}}\left(X_{\Gamma}\right)$ be the free abelian group generated by pairs $\left(D, g_{D}\right)$, where $D$ is a prime Weil divisor on $X_{\Gamma}$ and $g_{D}$ is a local equation for $D$ in a small neighborhood of $s$. The local equations give rise to local equations $g_{D}$ near $s$ for arbitrary Weil divisors $D$. Let $P_{\mathfrak{m}}\left(X_{\Gamma}\right)$ be the subgroup of pairs $\left(D, g_{D}\right) \in \operatorname{Div}_{\mathfrak{m}}\left(X_{\Gamma}\right)$ for which $D=\operatorname{div}(f)$ is the divisor of a meromorphic function $f$ satisfying

$$
f \cdot g_{D}^{-1}=1+O\left(q^{m}\right)
$$

near $s$. We define a generalized class group as the quotient

$$
\mathrm{Cl}_{\mathfrak{m}}\left(X_{\Gamma}\right)=\operatorname{Div}_{\mathfrak{m}}\left(X_{\Gamma}\right) / P_{\mathfrak{m}}\left(X_{\Gamma}\right) .
$$

It would be interesting to define suitable classes of special divisors in $\mathrm{Cl}_{\mathfrak{m}}\left(X_{\Gamma}\right)$ of arbitrary integral index $d$ and to prove a modularity result for the generating series of these classes.

\section{Acknowledgments}

We thank J. Funke, B. Gross, and S. Kudla for useful conversations on the content of this paper. Moreover, we thank the referee for his/her valuable comments.

\section{References}

[Alfes and Ehlen 2013] C. Alfes and S. Ehlen, "Twisted traces of CM values of weak Maass forms", J. Number Theory 133:6 (2013), 1827-1845. MR 3027941

[Borcherds 1998] R. E. Borcherds, "Automorphic forms with singularities on Grassmannians", Invent. Math. 132:3 (1998), 491-562. MR 1625724

[Borcherds 1999] R. E. Borcherds, "The Gross-Kohnen-Zagier theorem in higher dimensions", Duke Math. J. 97:2 (1999), 219-233. MR 1682249

[Bringmann et al. 2005] K. Bringmann, K. Ono, and J. Rouse, "Traces of singular moduli on Hilbert modular surfaces”, Int. Math. Res. Not. 2005:47 (2005), 2891-2912. MR 2192218

[Bruinier 2002] J. H. Bruinier, Borcherds products on $O(2, l)$ and Chern classes of Heegner divisors, Lecture Notes in Mathematics 1780, Springer, Berlin, 2002. MR 1903920

[Bruinier and Funke 2004] J. H. Bruinier and J. Funke, "On two geometric theta lifts", Duke Math. J. 125:1 (2004), 45-90. MR 2097357

[Bruinier and Funke 2006] J. H. Bruinier and J. Funke, "Traces of CM values of modular functions", J. Reine Angew. Math. 594 (2006), 1-33. MR 2248151

[Bruinier and Ono 2010] J. Bruinier and K. Ono, "Heegner divisors, $L$-functions and harmonic weak Maass forms", Ann. of Math. (2) 172:3 (2010), 2135-2181. MR 2726107

[Duke and Jenkins 2008] W. Duke and P. Jenkins, "Integral traces of singular values of weak Maass forms", Algebra Number Theory 2:5 (2008), 573-593. MR 2429454 
[Eichler and Zagier 1985] M. Eichler and D. Zagier, The theory of Jacobi forms, Progress in Mathematics 55, Birkhäuser, Boston, 1985. MR 781735

[Funke 2002] J. Funke, "Heegner divisors and nonholomorphic modular forms", Compositio Math. 133:3 (2002), 289-321. MR 1930980

[Gross 2012] B. H. Gross, "The classes of singular moduli in the generalized Jacobian", pp. 137-141 in Geometry and arithmetic, edited by C. Faber et al., EMS Ser. Congr. Rep., Eur. Math. Soc., Zürich, 2012. MR 2987658

[Gross et al. 1987] B. Gross, W. Kohnen, and D. Zagier, "Heegner points and derivatives of $L$-series, II”, Math. Ann. 278:1-4 (1987), 497-562. MR 909238

[Kim 2004] C. H. Kim, "Borcherds products associated with certain Thompson series", Compos. Math. 140:3 (2004), 541-551. MR 2041767

[Kudla 1997] S. S. Kudla, "Algebraic cycles on Shimura varieties of orthogonal type", Duke Math. J. 86:1 (1997), 39-78. MR 1427845

[Kudla 2003] S. S. Kudla, "Integrals of Borcherds forms”, Compositio Math. 137:3 (2003), 293-349. MR 1988501

[Kudla 2004] S. S. Kudla, "Special cycles and derivatives of Eisenstein series", pp. 243-270 in Heegner points and Rankin L-series, edited by Cambridge, Math. Sci. Res. Inst. Publ. 49, Cambridge Univ. Press, 2004. MR 2083214

[Kudla 2014] S. Kudla, "Another product for a Borcherds form”, preprint, 2014. arXiv 1402.0443

[Liu 2011] Y. Liu, "Arithmetic theta lifting and L-derivatives for unitary groups, II", Algebra Number Theory 5:7 (2011), 923-1000. MR 2928564

[McGraw 2003] W. J. McGraw, "The rationality of vector valued modular forms associated with the Weil representation”, Math. Ann. 326:1 (2003), 105-122. MR 1981614

[Serre 1988] J.-P. Serre, Algebraic groups and class fields, Graduate Texts in Mathematics 117, Springer, New York, 1988. MR 918564

[Zagier 1975] D. Zagier, "Nombres de classes et formes modulaires de poids 3/2", C. R. Acad. Sci. Paris Sér. A-B 281:21 (1975), Ai, A883-A886. MR 0429750

[Zagier 2002] D. Zagier, “Traces of singular moduli”, pp. 211-244 in Motives, polylogarithms and Hodge theory, I (Irvine, CA, 1998), edited by F. Bogomolov and L. Katzarkov, Int. Press Lect. Ser. 3, Int. Press, Somerville, MA, 2002. MR 1977587

Communicated by Richard Borcherds

Received 2015-08-27 Revised 2016-04-20 Accepted 2016-05-19

bruinier@mathematik.tu-darmstadt.de

Fachbereich Mathematik, Technische Universität Darmstadt, Schlossgartenstrasse 7, D-64289 Darmstadt, Germany

li@mathematik.tu-darmstadt.de Fachbereich Mathematik, Technische Universität Darmstadt, Schlossgartenstrasse 7, D-64289 Darmstadt, Germany 


\section{Algebra \& Number Theory}

msp.org/ant

\section{EDITORS}

MANAGING EDITOR

Bjorn Poonen

Massachusetts Institute of Technology

Cambridge, USA

\author{
EDITORIAL BOARD CHAIR \\ David Eisenbud \\ University of California \\ Berkeley, USA
}

BOARD OF EDITORS

$\begin{aligned} \text { Dave Benson } & \text { University of Aberdeen, Scotland } & \text { Susan Montgomery } & \text { University of Southern California, USA } \\ \text { Richard E. Borcherds } & \text { University of California, Berkeley, USA } & \text { Shigefumi Mori } & \text { RIMS, Kyoto University, Japan } \\ \text { John H. Coates } & \text { University of Cambridge, UK } & \text { Raman Parimala } & \text { Emory University, USA } \\ \text { J-L. Colliot-Thélène } & \text { CNRS, Université Paris-Sud, France } & \text { Jonathan Pila } & \text { University of Oxford, UK } \\ \text { Brian D. Conrad } & \text { Stanford University, USA } & \text { Anand Pillay } & \text { University of Notre Dame, USA } \\ \text { Hélène Esnault } & \text { Freie Universität Berlin, Germany } & \text { Victor Reiner } & \text { University of Minnesota, USA } \\ \text { Hubert Flenner } & \text { Ruhr-Universität, Germany } & \text { Peter Sarnak } & \text { Princeton University, USA } \\ \text { Sergey Fomin } & \text { University of Michigan, USA } & \text { Joseph H. Silverman } & \text { Brown University, USA } \\ \text { Edward Frenkel } & \text { University of California, Berkeley, USA } & \text { Michael Singer } & \text { North Carolina State University, USA } \\ \text { Andrew Granville } & \text { Université de Montréal, Canada } & \text { Vasudevan Srinivas } & \text { Tata Inst. of Fund. Research, India } \\ \text { Joseph Gubeladze } & \text { San Francisco State University, USA } & \text { J. Toby Stafford } & \text { University of Michigan, USA } \\ \text { Roger Heath-Brown } & \text { Oxford University, UK } & \text { Ravi Vakil } & \text { Stanford University, USA } \\ \text { Craig Huneke } & \text { University of Virginia, USA } & \text { Michel van den Bergh } & \text { Hasselt University, Belgium } \\ \text { Kiran S. Kedlaya } & \text { Univ. of California, San Diego, USA } & \text { Marie-France Vignéras } & \text { Université Paris VII, France } \\ \text { János Kollár } & \text { Princeton University, USA } & \text { Kei-Ichi Watanabe } & \text { Nihon University, Japan } \\ \text { Yuri Manin } & \text { Northwestern University, USA } & \text { Efim Zelmanov } & \text { University of California, San Diego, USA } \\ \text { Philippe Michel } & \text { École Polytechnique Fédérale de Lausanne } & \text { Shou-Wu Zhang } & \text { Princeton University, USA }\end{aligned}$

PRODUCTION

production@msp.org

Silvio Levy, Scientific Editor

See inside back cover or msp.org/ant for submission instructions.

The subscription price for 2016 is US $\$ 290$ /year for the electronic version, and $\$ 485 /$ year (+\$55, if shipping outside the US) for print and electronic. Subscriptions, requests for back issues and changes of subscribers address should be sent to MSP.

Algebra \& Number Theory (ISSN 1944-7833 electronic, 1937-0652 printed) at Mathematical Sciences Publishers, 798 Evans Hall \#3840, c/o University of California, Berkeley, CA 94720-3840 is published continuously online. Periodical rate postage paid at Berkeley, CA 94704, and additional mailing offices.

ANT peer review and production are managed by EditFLow ${ }^{\circledR}$ from MSP.

\section{PUBLISHED BY}

- mathematical sciences publishers

nonprofit scientific publishing

http://msp.org/

() 2016 Mathematical Sciences Publishers 


\section{Algebra \& Number Theory}

Volume $10 \quad$ No. $6 \quad 2016$

Modular elliptic curves over real abelian fields and the generalized Fermat equation $x^{2 \ell}+y^{2 m}=z^{p}$

SAMUELE ANNI and SAMIR SIKSEK

Geometry and stability of tautological bundles on Hilbert schemes of points

DAVID STAPLETON

Anabelian geometry and descent obstructions on moduli spaces

Stefan PATRIKIS, José Felipe Voloch and Yuri G. Zarhin

On the local Tamagawa number conjecture for Tate motives over tamely ramified fields JAY DAIGLE and MATTHIAS FLACH

Heegner divisors in generalized Jacobians and traces of singular moduli

JAN HENDRIK BRUINIER and YINGKUN LI

On 2-dimensional 2-adic Galois representations of local and global fields VYTAUTAS PAŠKŪNAS

A probabilistic Tits alternative and probabilistic identities

Michael LARSEN and ANER SHALEV 